\title{
Deployment of an Agent-based SANET Architecture for Healthcare Services
}

\author{
Christopher Chiu and Zenon Chaczko
}

\begin{abstract}
This paper describes the adaptation of a computational technique utilizing Extended Kohonen Maps (EKMs) and Rao- Blackwell-Kolmogorov (R-B) Filtering mechanisms for the administration of Sensor-Actuator networks (SANETs). Inspired by the BDI (Belief-Desire-Intention) Agent model from Rao and Georgeff, EKMs perform the quantitative analysis of an algorithmic artificial neural network process by using an indirectmapping EKM to self-organize, while the Rao-Blackwell filtering mechanism reduces the external noise and interference in the problem set introduced through the self-organization process. Initial results demonstrate that a combinatorial approach to optimization with EKMs and Rao-Blackwell filtering provides an improvement in event trajectory approximation in comparison to standalone cooperative EKM processes to allow responsive event detection and optimization in patient healthcare.
\end{abstract}

Keywords-BDI Agent Framework, Extended Kohonen Maps (EKM), healthcare infrastructures, Rao-Blackwell filtering, Sensor Actuator Networks (SANETs).

\section{Purpose of SANET Infrastructures}

$\mathbf{T}$ HE inspiration of using a distributed SANET infrastructure using biomimetic principles is the active monitoring of patients in health facilities. Traditional health communication models are reliant on passive identification systems, such as alert buzzers and pagers, to alert clinicians on the current status of a patient outside of intensive care. The necessity for an evolvable, self-healing network that encapsulates monitoring and control processes for a health care institution will augment existing healthcare practice by tracking the wellbeing of a patient into their recovery.

Healthcare middleware infrastructures are designed with a standardized exchange protocol and mechanism for interaction, such as HL7 [1], so it is logical that the design of the SANET infrastructure takes this in mind. The issue of efficient and concise data exchange for resource constrained systems, which can actively track and monitor changes in events is important as a quality attribute. Coupled with a meta- heuristic dynamic function using Extended Kohonen Maps for the neural network, a comparison is made on the benefits of optimization of neural networks to reduce noise introduced into the system. Therefore, the focus of this paper is to propose an systemic infrastructure suitable for SANET environments [2], [3], with an algorithmic foundation based on Rao-Blackwell-Kolmogorov optimization in the Sun Java environment.

The SANET healthcare infrastructure model must be designed with hard real-time criticality in mind, while also

C. Chiu and Z. Chaczko are with the Faculty of Engineering, University of Technology, Sydney, Australia (e-mails: christopher.chiu@uts.edu.au, zenon.chaczko@uts.edu.au).
TABLE I

QUALITY ATTRIBUTES OF COSA-BASED INFRASTRUCTURES

\begin{tabular}{ll}
\hline \hline Attribute & Description \\
\hline Feature & $\bullet$ Utilizing an Extended Kohonen Map (EKM) \\
Mapping & by Kohonen [4], [5], the self-organizing map \\
& partitions sensory-aware spaces discretely; thus the \\
& generalization capability arises from its self- \\
& organization during map training [6]. \\
& Map resolution is improved in frequently \\
& encountered stimuli regions, thus mimicking \\
& biological sensory perceptions where reinforced \\
& practice allows for prediction of anticipated events.
\end{tabular}

Multi- $\quad$ Formulated as a non-linear multi-variate regression

variate

Regression

Techniques problem [7]-[9], the main issue is that training samples must be regularly collected for error sampling rates. The sampling process is simplified by providing qualitative feedback at the end of the executing control sequence [9]. This technique ensures smoothness when local minima or maxima results are encountered, such that sensor variations are minimized.

Data - The filtration process is coupled with multivariate Filtration regression to eliminate noise within the system Process process [10]. This allows for the systemic transition from a global awareness of reaching the target objective from within the local domain knowledge space. This allows for a further refining process of the data collection model and enhances the final result of the regression algorithmic samples.

Multi- - Reinforcement learning heuristics, based upon Objective non-deterministic biological concepts, provides a best Meta- effort optimum routing and clustering organization Heuristics solution depending on the current situation [10]. The feedback loop is closed to ensure that knowledge from the environment is retained, such that the predictive models that determine future clustering and routing paths can represent system state behavior and concurrent data repositories.

being fault-tolerant and incorporate redundancy to ensure the data responses and feedback is delivered within finite limits. Thus, the infrastructure must achieve strict Quality of Service (QoS) qualitative requirements [11], [12], inclusive of scalability, robustness, security, privacy and efficiency. The SANET infrastructure should support the process of software development by facilitating integration of components and reducing the accidental complexities related to heterogeneous environments in health institutions. The Cooperative-Oriented Sensors Actuators model (COSA) by Chaczko [13] is used to represent a SANET neighborhood and its moderation by two global aspects: the accuracy of a node's position and the node's immunity to error propagation. The algorithmic categories are 
TABLE II

INTERCONNECTIVITY BETWEEN HOLONIC COMPONENTS

\begin{tabular}{ll}
\hline \hline Connection & Component Type \\
\hline $\begin{array}{l}\text { Connector Facilities } \\
\text { within Components }\end{array}$ & $\begin{array}{l}\text { • The remote procedure calls or remote } \\
\text { method invocation between agent nodes or } \\
\text { cluster heads. }\end{array}$ \\
$\begin{array}{l}\text { Horizontal Models } \\
\text { of Infrastructure }\end{array}$ & $\begin{array}{l}\text { • Request brokers or publish-subscribe } \\
\text { mechanisms between sensory components } \\
\text { in same platform. }\end{array}$ \\
$\begin{array}{l}\text { Vertical Models } \\
\text { of Domain Paradigm }\end{array}$ & $\begin{array}{l}\text { — Common semantics and context } \\
\text { awareness, and high-level reusable } \\
\text { services for multiple platforms for sensor } \\
\text { and actuator control. }\end{array}$ \\
\hline \hline
\end{tabular}

ordered according from local to global domain concerns in Table I.

\section{A. Basis of BDI-based Holonic Infrastructures for SANETs}

The biomimetic approach used in the experimental model is based on the need for SANET architectures to manage infrastructures on a distributed level. Large-scale infrastructures such as healthcare monitoring are an ideal candidate for the holonic model, as service orientation is focused on a patient-centric environment [12], [14], hence the motivation of utilizing a BDI-based agent holonic infrastructure to model the patient's needs and concerns as an individual agent-based holon. The agent's desires and intentions are captured and realized within the holon entities in practice, so that the sensory determination is executed in a real-time task control process. In Figure 1, distributed data space concerns are addressed through Tuple Space [15] implementations that need to be supported in a SANET component middleware system, such as how a patient's location can be notified by the doctor or next of kin, depending on the current patient's status. Holonics follows the multiverse concepts of elemental slicing of a core entity of representing the structures and interconnections between internal entities [14] elaborated in Table II.

In Table III and Figure 2, the biomimetic model perspective can be projected onto a $3 \mathrm{D}$ space based on the POE Classification Model [14], [16]. The POE model represents the different levels of organization, with POE standing for Phylogeny, Ontogeny and Epigenesis.
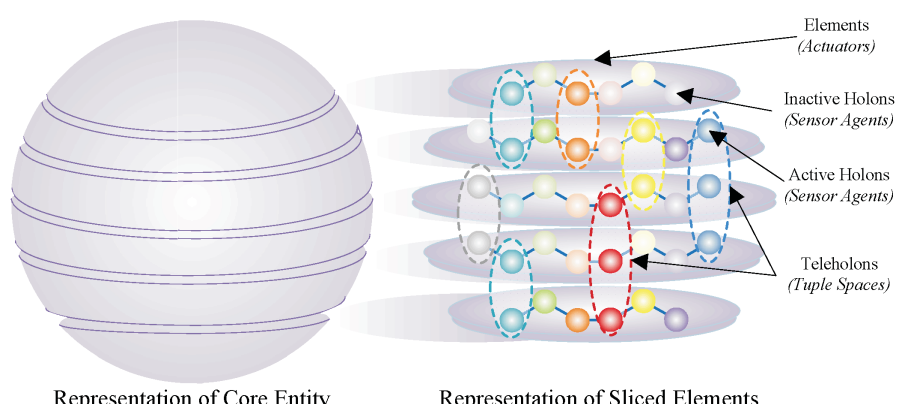

Fig. 1. Tuple space paradigm consists of distributed Memory Spaces overlaid on multiple actuator platforms [14].
TABLE III

POE MODEL AND SANET CORRELATIONS

\begin{tabular}{|c|c|}
\hline Dimension & Context and Correlation \\
\hline Phylogeny & $\begin{array}{l}\text { - Biological Context: Entail evolution of species } \\
\text { genetics. } \\
\text { - SANET Correlation: This relates to the } \\
\text { implementation of heuristic tracking algorithms } \\
\text { (Cooperative EKMs) for an individual surgical } \\
\text { training exercise. }\end{array}$ \\
\hline Ontogeny & $\begin{array}{l}\text { - Biological Context: Concerned with cellular growth } \\
\text { process, multi-cellular organization, cellular division } \\
\text { and differentiation from the parent to child cells. } \\
\text { Each child cell processes a copy of the original } \\
\text { genome. } \\
\text { - SANET Correlation: The perspective of calculating } \\
\text { EKM activation energies for the simulator to } \\
\text { provide feedback to the trainee. }\end{array}$ \\
\hline Epigenesis & $\begin{array}{l}\text { - Biological Context: This involves the adaptation and } \\
\text { learning processes. The nervous, immune and } \\
\text { endocrine systems are characterized by epigenesis. } \\
\text { - SANET Correlation: This space corresponds to the } \\
\text { facets or aspects of specialist trainee feedback by } \\
\text { responding to stimuli and weights adaption feedback. }\end{array}$ \\
\hline
\end{tabular}

\section{DOMAIN ANALYSIS OF SANETS}

\section{A. Cooperative EKMs with R-B Optimization}

Procedural controls are formed as a discrete set of commands to be used by reinforcement learning algorithms [5] to be selected from a library of heuristic functions for a distributed network in a healthcare concern. Reinforcement learning is driven by continuous control space functions [4], as indirect-mapping methods provide fluid decision choices than direct mapping. The accuracy in sensory stimuli control is important where external factors directly affect the network's robustness and reliability, combined with Rao-Blackwell optimization, allows for developing a feature map approach using co-operative EKMs with indirect mapping to improve the responsiveness of the tracking process [4], [10].

Event predication can be achieved using linear trajectory models in Euclidean space [9]. However, multi-dimensional problems outside of Euclidean space are sacrificed as a result, inclusive of network interference and ensuring energy efficiency. By not factoring the domain concern of including the problem set into a singular process model, the problem scope increases exponentially [14]. The solution is to transform the views and decompose them into a singular map of the highest activation energies or stimuli, which is achieved once the maximum value of the integration matrix is calculated for all EKMs for each of the input stimuli received from the

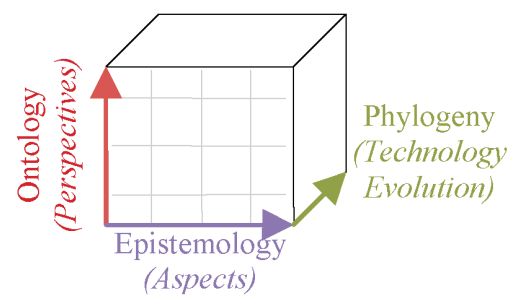

Fig. 2. POE classification (phylogeny, ontology, epistemology) [15], [16]. 
TABLE IV

EXTENDED KOHONEN MAPPING TECHNIQUES

\begin{tabular}{|c|c|}
\hline $\begin{array}{l}\text { Mapping } \\
\text { Method }\end{array}$ & Mapping Methodology \\
\hline $\begin{array}{l}\text { By Direct } \\
\text { Mapping } \\
\text { Method }\end{array}$ & $\begin{array}{l}\text { - Node inputs are mapped to sensory stimuli, with } \\
\text { indirect-maps of sensory stimuli linked to a node } \\
\text { with the utilization of control parameters, including } \\
\text { energy discharge, signal strength and node roles as } \\
\text { ordinary node, cluster head or location anchors. }\end{array}$ \\
\hline $\begin{array}{l}\text { By } \\
\text { Indirect- } \\
\text { Mapping } \\
\text { Method }\end{array}$ & $\begin{array}{l}\text { - Continuous sensory stimuli space is mapped to the } \\
\text { node; instead of direct-mapping that map } \\
\text { continuous sensory stimuli space to the actual } \\
\text { directives. Quality evaluators determine optimum } \\
\text { clustering using Rao-Blackwell optimization [13] } \\
\text { criteria. }\end{array}$ \\
\hline
\end{tabular}

SANET's environment. Insufficient sensory controls can result in unexpected or undesirable outcomes, leading to possible faults in navigating a route to the gateway [4], [5]. When EKMs are established in the weighted-sum ensemble, a similar problem of unsolvable outcomes also takes place. This can be solved by using an indirect EKM mapping method in conjunction with Rao-Blackwell Optimization, whereby the control vector is manipulated indirectly via a control parameter space [4].

The environmental concern shown in Figure 3: Co-operative EKM Process [10]or a given SANET domain can be summarized in the following statement tasks:

- For initial state described by input vector $u(0)$ in input space $\mathrm{U}$, inclusive of the sensory perception;

- Adapt sequence of control vectors $\mathrm{c}(\mathrm{t}), \mathrm{t}=0$. . .t- 1 , in sensor control space $\mathrm{C}$ and solve activation energies;

- With resultant goal state elaborated by $\mathrm{u}(\mathrm{T}) \in \mathrm{U}$ that adapts the structure for a desired objective, thus reacting to the stimulus and changing the input state.

- Perform Rao-Blackwell optimization on the weighted values, with the final estimator is used for evaluating effectiveness of the neuron weights.

Using a multi-objective approach with co-operative EKMs as the modeling function has been adopted, with a reinforcement learning technique chosen on the basis of the situation or context [4]. Interleaved EKMs that cooperate and compete to self-organize can enable a node to optimize in managing clustering and routing dynamically, whereby the node's output control is less than the total variable control available. Euclidean and weighted-sum ensemble methods have detrimental outcomes, even though a continuous sensory control space is implemented [9].

\section{B. Multi-Objective Meta-Heuristic Algorithm (MMA)}

The paradigm of meta-heuristics is taking the optimum yielded results of selected heuristic methods that are suitable for the problem domain in a healthcare environment. In particular, nature-inspired functions are most suitable when considering the learning capability of the healthcare network that is suitable for patient prediction models. Unlike with trajectory functions, which are concerned with local thresholds to yield a result, bio-inspired functions take a holistic view
TABLE V

POE-STAGED EXPERIMENTAL PROCESS

\begin{tabular}{ll}
\hline \hline POE Stage & Experimental Process \\
\hline Stage 1: Model & • This stage is about performing an Unsupervised \\
Domain & Learning Heuristic on the environment. This \\
Environment & involves modeling the environmental concerns to \\
(Phylogenical) & $\begin{array}{l}\text { determine how the data should be organized for } \\
\text { optimum clustering and routing mechanisms. } \\
\end{array}$ \\
& $\begin{array}{l}\text { EKMs current experiment considers the use of } \\
\text { the input sensory perception at the beginning. }\end{array}$
\end{tabular}

Stage 2: Train for Environment (Ontological)

- This stage is about performing a Reinforcement Learning Heuristic. This allows the environment to be aware of its context, and learn from its own experience and that of its cooperative actors. - The experiment incorporates genetic algorithmic heuristics to achieve learning capability. Classical techniques such as brute-force analysis is computationally inefficient, and is infeasible to consider all outcomes that lead to an optimum solution, only the best-effort solution in time.

Stage 3: Refine - This stage is about factorizing the Training Set. Training Set (Epigenetical) This optimizes the training set for a given learning heuristic to a singular vector, reducing invariability for long-term forecasting. - The current experiment considers generalized optimization techniques to reduce noise and other variability in the training set. This is necessary to select an ideal candidate that establishes the fitness condition for the SANET environment, such as maintaining the minimum energy condition and optimizing bandwidth.

of the dynamic, evolving system. In essence, by combining a multi-dimensional approach to analyzing a given dataset, we can enhance the regenerative learning capability for any given heuristic optimization model. Current experiments for SANET modeling examine genetic algorithms and Particle Swarm Optimization (PSO) [17] as they both implicit-based reinforcement learning methods that attempt to seek a long term advantage through a representation set of scalar rewards. A filtration process is then applied to the mapping data to clear erroneous and randomized data introduced as a result of the algorithmic calculations, allowing for a clearer weighting adaption once a response is made to the stimuli.

The application of the heuristic algorithm follows the approach of applying EKM principles with R-B optimization to the problem domain in SANETs [13], [17]. This requires the modeling map to be translated into a training vector to be processed against a scalar rewards vector, which reflects the optimum energy or routing condition; depending on the current environmental concerns of the SANET network.

The number of evolutions to determine an acceptable result will affect the quality of the process, but there requires a balance between obtaining a quality evaluation, which requires more processing time and is power intensive, with minimizing the time to process the heuristic function for resource conservation. The implementation method for metaheuristic functions in the experimental model is established procedurally in Figure 4 and Table V. 


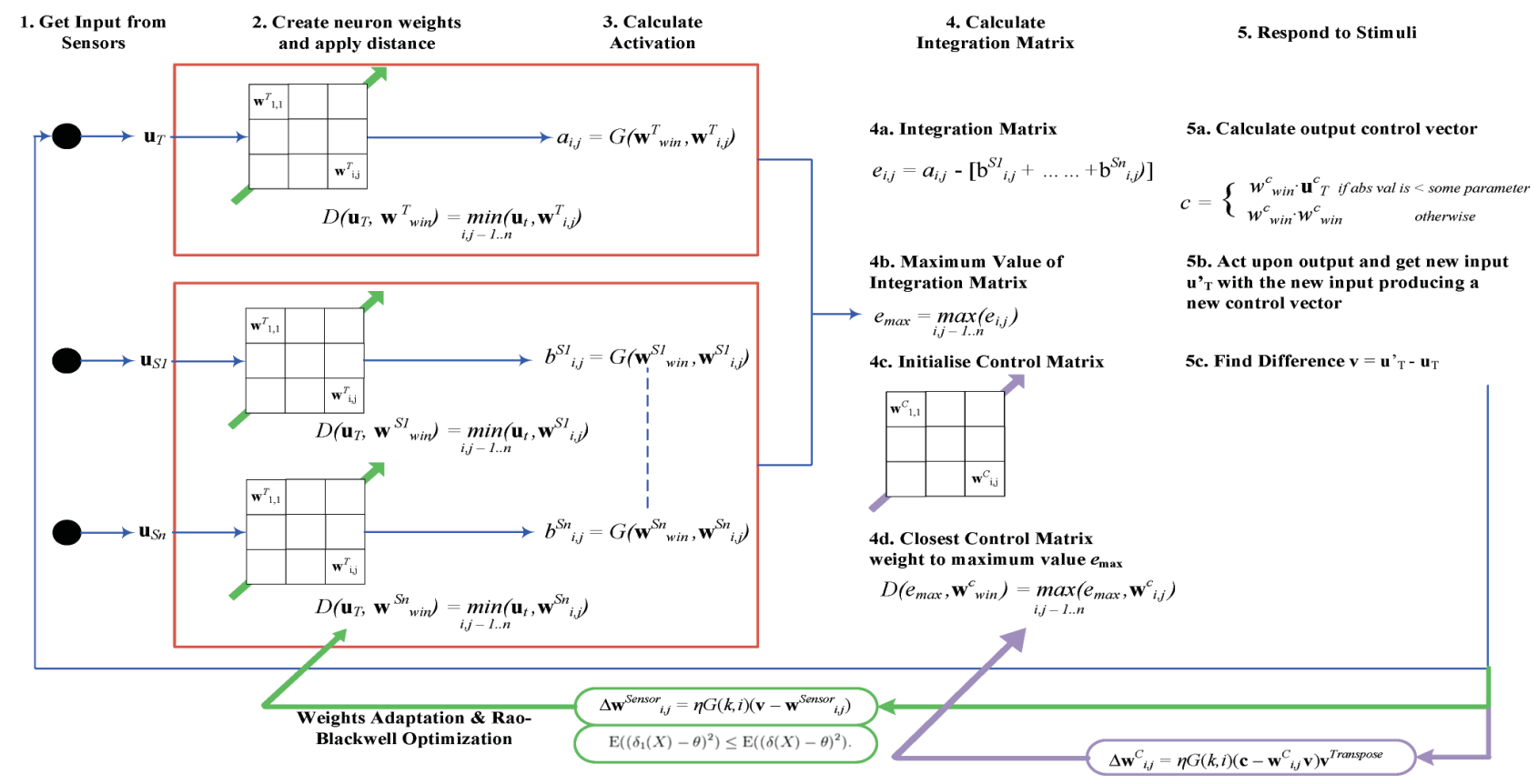

Fig. 3. Co-operative EKM Process [10].

\section{EXPERIMENTAL MODEL}

The evaluation of co-operative EKMs with R-B optimization on SANETs is executed using the framework conceived by Chaczko, et al [10]. The Jadex framework by University of Hamburg, shown in Figure 5: Jadex Simulation Environment [13] allows for convenient monitoring and tracking of SANET events by pre-assigning event trajectories in the network field in an interactive manner.

\section{A. General Assumptions}

- The topology of the SANET surgical training system is two-dimensional, so altitude is constant and negligible between nodes.

- All nodes are powered at $100 \%$ capacity. Energy dissipation is calculated using inverse square law.

- The event trajectory is not predefined; so start and end points are calculated randomly using Fast MersenneTwister method.

- The network is considered to be interference free; as such RF communication is not evaluated in the experimental model.

\section{B. Experimental Method}

The experiment is completed with the methodology by establishing the following simulation constraints:

1) A population of $n$ nodes is distributed randomly via Fast Mersenne-Twister, in a two-dimensional network of $100 \mathrm{~m} \times 100 \mathrm{~m}$. Node populations tested include:
a) 100 ;
b) 250 ;
c) 500 ;
d) 1000 ;

e) 2000;

f) 3000 ;

g) 4000

h) 5000 .

2) An event trajectory is executed from a point in the network area; of which the test path course is either:

- Linear Path: A linear path consists of an event trajectory where the entry and exit point from the area is of constant gradient.

- Arc-formation Path: Arc-formation consists of an event trajectory where the entry and exit point will either be increasing or decreasing in gradient, forming a circle segment.

- Pseudo-random Path: A pseudo-random path using the Mersenne-Twister method combines 2(a) and 2(b) at various points throughout the trajectory, until it reaches the exit point.

3) The algorithm selects the route from the node in range of the approximate trajectory to be established to the sink; such that the closer the algorithm is to calculating the event path, the more optimum the route will be to establish successful negotiations.

- Co-operative EKMs with Particle Swarm Optimization (PSO): Co-operative EKMs use an indirectmapping SOM map to train the control parameters in which to converge at the final trajectory point; in such a fashion to actively train the neural network to seek positive outcomes to determine a route to the sink.

- Co-operative EKMs with R-B Optimization: In conjunction with Co-operative EKMs, a filtrating mechanism is applied to the weights adaptation map using 


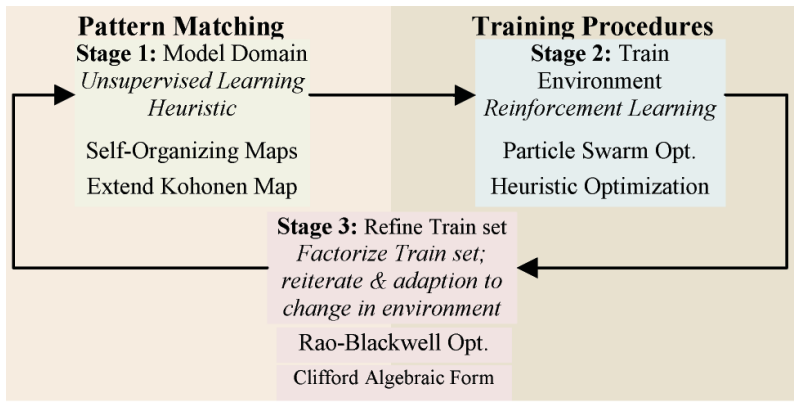

Fig. 4. Multi-objective meta-heuristic process [10].

the Rao-Blackwell optimization method to assist in the event tracking functions.

In conjunction, the reinforcement learning heuristic applied to the SOM map as a training vector set, and a vector test set is applied to maintain accuracy of path estimation. Using Particle Swarm Optimization [17], the shortest Euclidean distance between the specific trajectory point and the nearest node is nominated within the routable path to the sink or health repository.

4) The experiment is executed for 1000 iterations to calculate the mean rate of identification of the trajectory's target point, when the event exits the area:

- A maximum margin of error is a $2 \mathrm{~m} \times 2 \mathrm{~m}$ area where final approximate point is found.

- A successful identification is where the final endpoint is within a $95 \%$ confidence interval of the entire SANET network. Any estimation outside of this threshold is identified as failed.

\section{EXPERIMENTAL RESULTS}

The results demonstrate that in comparison to co-operative EKMs with PSO and Rao-Blackwell optimization, the final results are positive when pseudo-random trajectory tracking is required. While standard Co-operative EKMs perform adequately in the given scenarios; co-operative EKMs with RaoBlackwell optimization demonstrates a perceptible improvement in the identification rate over standard co- operative EKM algorithms. This is evident with a greater node population, as the granularity of determining a nearest neighboring node to

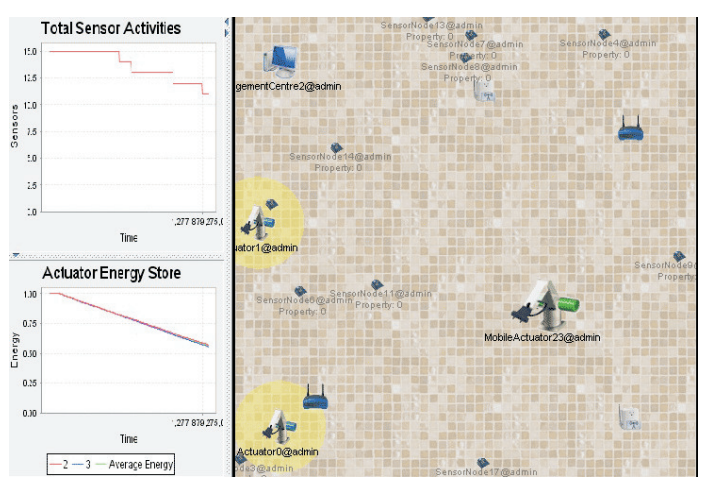

Fig. 5. Jadex simulation environment [13].

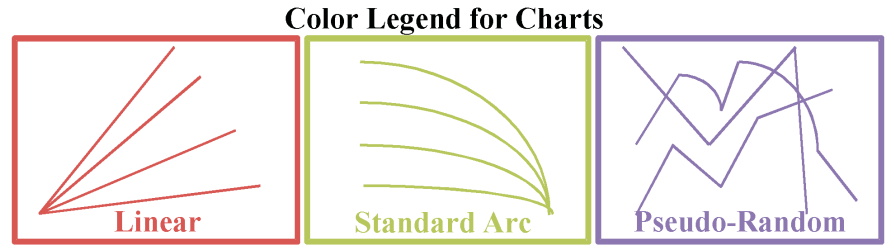

Co-operative EKMs
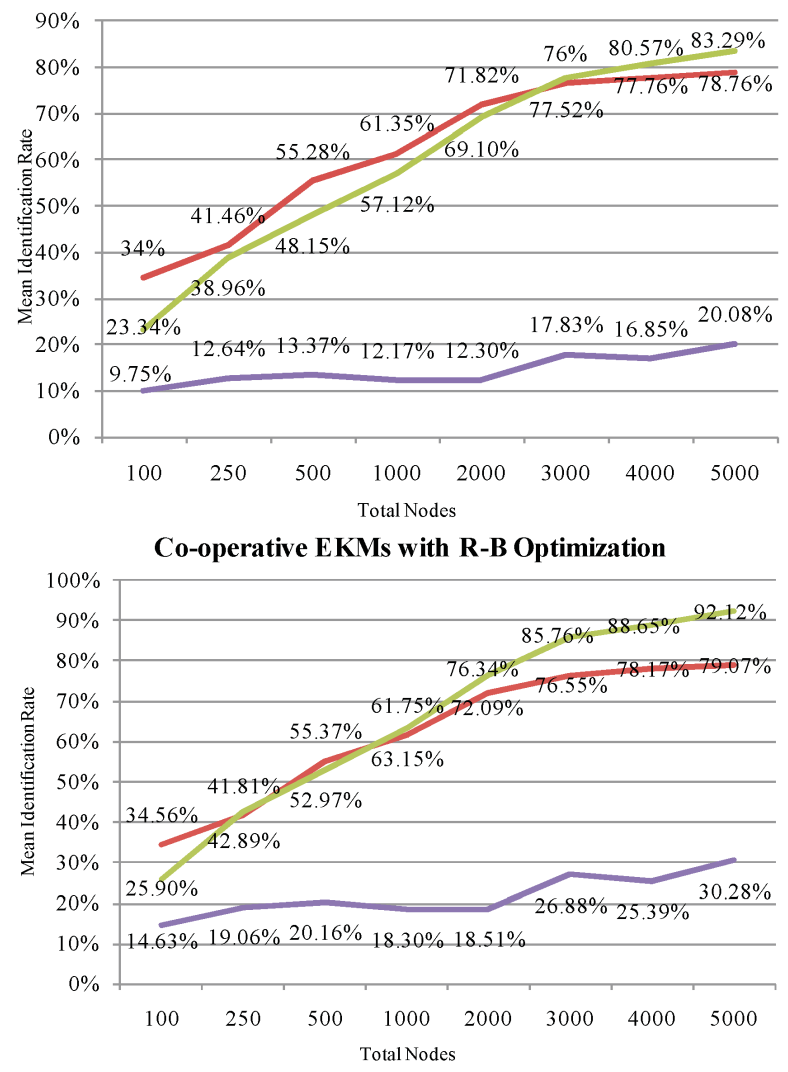

Fig. 6. Experimental results of detection success.

route is reduced for the fixed size of the network. As shown in Figure 6: Experimental Results of Detection there is a 50\% relative improvement in the average mean detection rates with Rao-Blackwell optimization for the pseudo-random trajectory compared to standard co-operative EKMs. As co-operative EKM with R-B optimization demonstrate improvement over passive learning techniques, current prediction rates are sufficient for routing estimation capability, which indicate the process of refining the training set for remodeling is necessary to improve routing conditions.

The analysis of co-operative EKMs as shown below in Table VI and VII, when assessed in terms of performance of pseudorandom tracking, requires more analysis into the algorithmic procedure. In particular, the thresholds established for determining positive or negative learning reinforcement is an issue that needs to be evaluated for an in-depth assessment. The tolerance levels used to calculate the thresholds is important, as subtle variations in tolerance may yield undesirable results. As a case in point, reducing tolerance levels too far will result in the inflexibility of the algorithm to adapt to changes the event trajectory; the corollary is that generous tolerance levels 
TABLE VI

COMPARATIVE ASSESSMENT AND EVALUATION CO-OPERATIVE EKMS WITH PSO

\begin{tabular}{ll}
\hline \hline Assessment & Evaluation \\
\hline Quantitative & $\bullet$ Demonstrates an indirect-mapping EKM can \\
pssessment & $\begin{array}{l}\text { provide detection to optimize for local obstruction } \\
\text { and global target identification concerns in } \\
\text { a distributed sensor space. }\end{array}$ \\
Qualitative & • Results show a smoother tracking mechanism to \\
Assessment & $\begin{array}{l}\text { monitor events in real-time, compensating for } \\
\text { random events [4], [6]. } \\
\end{array}$ \\
& $\begin{array}{l}\text { with an enhanced training set refinement } \\
\text { mechanism, yields more consistent results as due } \\
\text { to an optimal training vector }\end{array}$ \\
\hline \hline
\end{tabular}

will yield undesirable tracking results when noise or faulty nodes produce invalid data.

Furthermore, the use of Clifford algebraic forms can be used in a SANET system in terms of extending the geometric form of the network's geometric structure [9], [18]. The quaternions of the network can be extended for 4 dimensional rotation groups, which serves as an ideal base for energy conservation and core attributes of a SANET network. Although the current simulation framework does not incorporate Clifford algebraic forms as an optimization technique, the modular design of the simulation environment will enable incorporation of this technique in the next iteration of the healthcare framework.

As a consequence, the potential of Co-operative EKMs with R-B optimization to identify events within a SANET network is evident; but as with all passive learning heuristic methods, a heuristic ensemble approach using MMA is necessary to train the algorithm to evaluate and determine the tolerance thresholds that are most suitable for the current conditions. The implementation of co-operative EKMs with alternative heuristic algorithms such as genetic algorithms will need to be considered in future to evaluate improvement in the mean identification rate. Furthermore, a feedback mechanism incorporating training set factorization is required to minimize error rates and ensure a consistent set of data outcomes, and allow for more fluid responses in the routing and tracking network functions.

\section{Analysis of Results}

\section{A. Hybrid Holonic Infrastructure Domain Mapping}

The consideration of the Holonic Hybrid infrastructure corresponds with the needs of the healthcare infrastructure, with holons represented as individual resource concerns of which the holonic infrastructure encapsulates the pool of shared resources for the entire network, and the teleholons represented as the collaboration or synchronization between resource holons. As such, it addresses healthcare management from two central views:

- Holonic Concerns: Horizontal perspectives for a single cluster; such as the individual concerns and status of a patient in the care of a clinician in charge.

- Teleonic Concerns: Vertical perspectives for clusters and external networks, such as the facilitation and negotiation
TABLE VII

COMPARATIVE ASSESSMENT AND EVALUATION

CO-OPERATIVE EKMS WITH R-B OPTIMISATION

\begin{tabular}{ll}
\hline \hline Assessment & Evaluation \\
\hline Quantitative & • Shows that the filtration method can yield \\
Assessment & $\begin{array}{l}\text { a noticeable improvement of 50\% in detection rates } \\
\text { for randomized routes. }\end{array}$ \\
& $\begin{array}{l}\text { - The improvement in detection success is a result of } \\
\text { the Rao-Blackwell estimator used when optimizing } \\
\text { the neural weights, compensating noise introduced. }\end{array}$ \\
& $\begin{array}{l}\text { Q Cooperative EKMs do not adjust to interference or } \\
\text { Qualitative }\end{array}$ \\
& $\begin{array}{l}\text { noise introduced into the system, such that the final } \\
\text { poor results. }\end{array}$ \\
& $\begin{array}{l}\text { - Results show that Co-operative EKMs combined } \\
\text { with R-B Optimization improves the tracking } \\
\text { process by reducing noise and interference within } \\
\text { the feedback loop [14], [15]. }\end{array}$ \\
\hline \hline
\end{tabular}

of health records between wards or specialist units, as when a patient handover takes place between staff shifts.

\section{B. Cooperative EKMs with R-B Optimization Validation}

The notable variability in the identification rate indicates a need to improve the quality of training mechanisms to reinforce positive selection processes, so the aggregation of the final routing selection is optimal for the system environment. Furthermore, the implementation of refining the training set is necessary to ensure that the SANET modeling functions do not introduce internally generated erroneous data that will impact on the system's ability to make future predictions based on historical trends. The relative improvement of $50 \%$ on traditional cooperative EKMs is significant to note; with the aim being a yield in the trajectory projection to ensure results are within the $95 \%$ confidence interval in tracking projections. In particular, further examination is required to determine the trade-off between yielding an efficient route which will be more computationally intensive, or a best-effort route that will be more energy conservative. However, the distributed nature of SANETs means that computational calculations can take place for each routing hop, so that while the local calculations are considered for each route, a global picture is established for the entire route to the gateway or central healthcare repository.

\section{CONCLUSION}

The method of adaptive SANET management for healthcare monitoring with co-operative EKMs coupled with RaoBlackwell optimization functions is beneficial, with the current results demonstrating indirect-mapping EKM generating more proficient routing and clustering conditions when compared to direct-mapping EKMs. Furthermore, the control parameters of the indirect-mapping EKM can be enhanced with RaoBlackwell algorithms to allow convergence and better optimization, with choices in the reinforcement learning technique providing context-aware outcomes to improve the reinforcement learning capability for patient monitoring in health institutions. 


\section{REFERENCES}

[1] Health Level Seven (HL7) Inc, http://www.hl7.org/, Last Visited 22nd January 2010.

[2] C. C. Chiu, Z. Chaczko, and P. Moses, "Advanced Extended Kohonen Mapping Modelling Techniques for Sensor Actor Networks," in 4th International Conference on Broadband Communication, Information Technology \& Biomedical Applications (BroadBandCom 2009), Wroclaw, Poland, July 2009, pp. 15-18.

[3] _ _ "Sensor Actor Network Modeling Utilizing the Holonic Architectural Framework," International Journal of Electronics and Telecommunications, vol. 55, no. 4, 2009.

[4] K. H. Low, W. K. Leow, and M. H. Ang Jr., An Ensemble of Cooperative Extended Kohonen Maps for Complex Tasks In Neural Computation. MIT Press, 2005, vol. 17.

[5] J. del R. Mill'an, D. Posenato, and E. Dedieu, "Continuous-Action Qlearning," Machine Learning, pp. 249-265, 2002.

[6] A. J. C. Sharkey and N. E. Sharkey, "Combining Diverse Neural Nets," Knowledge Engineering Review, pp. 231-247, 1997.

[7] L. P. Kaelbling, M. L. Littman, and A. W. Moore, "Reinforcement Learning: A Survey." Journal of Artificial Intelligence Research, vol. 4, pp. 237-285, 1996.

[8] T. Kohonen, Self-Organizing Maps, 3rd ed. New York: Springer-Verlag, 2000.

[9] H. B. Lawson and M. L. Michelsohn, Spin Geometry. Princeton, NJ: Princeton University Press, 1989.

[10] Z. Chaczko and C. Chiu, "Cooperative EKMs for Wireless Sensor Networks," Eurocast, vol. 12, pp. 304-305, 2009, published by Gran Canarias Las Palmas University Press.

[11] Z. Chaczko, D. Davis, and V. Mahadevan, "New Perspectives on Teaching and Learning Software Systems Development in Large Groups," in Proceedings of the FIfth International Conference on Information Technology Based Higher Education and Training, Istanbul, Turkey, 2004.
[12] C. Szyperski, "Emerging Component Software Technologies - A Strategic Comparison," Software - Concepts and Tools, vol. 19, no. 1, pp. 2-10, 1998.

[13] Z. Chaczko, J. Nikodem, R. Klempous, and M. Nikodem, "Sensors Localisation Methods in the COSA Framework," in AusWireless'07: The 2nd International Conference on Wireless Broadband and Ultra Wideband Communications, 2007, p. 60.

[14] Z. Chaczko, V. Mahadevan, and J. Nikodem, "A Bio-Inspired Telecollaboration Service Taxonomy: Usability Related Concerns," in Third International Conference on Broadband Communications, Information Technology \& Biomedical Applications, 2008, pp. 209-214.

[15] Z. Chaczko et al, "NICE Models of Biomimetic Software Systems," in CSIRO ICT Centre Conference, 2007.

[16] M. Sipper, E. Sanchez, D. Mange, M. Tomassini, Perez-Uribe, and A. Stauffer, "A Phylogenetic, Ontogenetic, and Epigenetic View of Bio-Inspired Hardware Systems," IEEE Transactionson Evolutionary Computation, vol. 1, pp. 83-97, 1997.

[17] M. Clerc, Particle Swarm Optimization: ISTE (International Scientific and Technical Encyclopedia). Hermés Science, 2005, translated from L'optimisation par essaims particulaires. Versions paramétriques et adaptatives.

[18] P. Lounesto, Clifford Algebras and Spinors. Cambridge: Cambridge University Press, 2001.

[19] J. Ghosh and A. Nag, "An Overview of Radial Basis Function Networks," in Radial Basis Function Networks: New Advances in Design, R. J. Howlett and L. C. Jain, Eds. New York: Physica-Verlag, 2001, pp. 1-36.

[20] K. H. Low, W. K. Leow, and M. H. Ang Jr., "Task Allocation via SelfOrganizing Swarm Coalitions in Distributed Mobile Sensor Network," in Proceedings of 19th National Conference on AI (AAAI-04), 2004, pp. 28-33. 